A. A. Kilin

\title{
Optimal programming of the rigid body dynamics problems
}

Vestnik Udmurtskogo Universiteta. Matematika. Mekhanika. Komp'yuternye Nauki, 2008, вып. 3, 126-135

\section{References}

[1] Arkhangelskii Yu. A., Dinamika bystrovraschayuschegosya tverdogo tela, Nauka, M., 1985 Mathscinet

[2] Barkin Yu.V., "Periodicheskie i uslovno-periodicheskie resheniya $\mathrm{v}$ zadache o dvizhenii tyazhelogo tverdogo tela vokrug nepodvizhnoi tochki", Prikl. Mat. Mekh., 45:3 (1981), 535-544 MathSciNet Zentral MÄTH

[3] Borisov A. V., Mamaev I. S., Dinamika tverdogo tela, RKhD, Moskva, Izhevsk, 2001, 384 pp. MathseiNet

[4] Borisov A. V., Simakov N. N., "Bifurkatsii udvoeniya perioda v dinamike tverdogo tela", Reg. i khaot. dinam., 2:1 (1997), 64-75 MathsciNet

[5] Gorr G. V., "Ob odnom dvizhenii tyazhelogo tverdogo tela v sluchae GoryachevaChaplygina", Prikl. mat. mekh., 34:6 (1970), 1139-1143

[6] Gorr G. V., Kudryashova L. V., Stepanova L. A., Klassicheskie zadachi dinamiki tverdogo tela, Naukova dumka, Kiev, 1978, 296 pp.

[7] Gorr G.V., Levitskaya G.D., "Ob odnom periodicheskom dvizhenii giroskopa Goryacheva-Chaplygina", Mekh. tv. tela, 1971, no. 3, 101-106

[8] Gorr G.V., Savchenko A. Ya., "Ob odnom periodicheskom dvizhenii v reshenii S. V. Kovalevskoi", Mekh. tv. tela, 1971, no. 3, 64-69

[9] Delone N.B., "K voprosu o geometricheskom istolkovanii integralov dvizheniya tverdogo tela okolo nepodvizhnoi tochki, dannykh S. V. Kovalevskoi", Mat. sbornik Kruzhka lyubitelei mat. nauk, 16:2 (1892), 346-351

[10] Kozlov V.V., Metody kachestvennogo analiza $v$ dinamike tverdogo tela, Izd-vo RKhD, Izhevsk, 2000, 256 pp.

[11] Chenciner A., Montgomery R., "A remarkable periodic solution of the three body problem in the case of equal masses", Annals of Mathematics, 152 (2000), 881-901 dol MathsciNet ZentralmATH

[12] Galgani L., Giorgilli A., Strelcyn J.-M., "Chaotic motions and transition to stochasticily in the classical problem of the heavy rigid body with a fixed point", Nuovo Cimento B, 61:1 (1981), 1-20 dol MathsciNet ads*

[13] Simó C., "Dynamical properties of the figure eight solution of the three-body problem", Celestial Mechanics, dedicated to Donald Saari for his 60th Birthday, Proceedings of an International Conference on Celestial Mechanics (15-19 December, 1999 at Northwestern University, Evanston, Illinois), Contemporary Mathematics, 292, American Mathematical Society, Providence, RI, 2002, 209 MathsciNet ZentrallMÄH ads* 